\title{
Gravitational production of dark matter during reheating
}

\author{
Yann Mambrini $\circledast^{1, *}$ and Keith A. Olive ${ }^{2, \dagger}$ \\ ${ }^{1}$ Université Paris-Saclay, CNRS/IN2P3, IJCLab, 91405 Orsay, France \\ ${ }^{2}$ William I. Fine Theoretical Physics Institute, School of Physics and Astronomy, University of Minnesota, \\ Minneapolis, Minnesota 55455, USA
}

(Received 23 February 2021; accepted 11 May 2021; published 7 June 2021)

\begin{abstract}
We consider the direct $s$-channel gravitational production of dark matter during the reheating process. Independent of the identity of the dark matter candidate or its nongravitational interactions, the gravitational process is always present and provides a minimal production mechanism. During reheating, a thermal bath is quickly generated with a maximum temperature $T_{\max }$, and the temperature decreases as the inflaton continues to decay until the energy densities of radiation and inflaton oscillations are equal, at $T_{\mathrm{RH}}$. During these oscillations, $s$-channel gravitational production of dark matter occurs. We show that the abundance of dark matter (fermionic or scalar) depends primarily on the combination $T_{\max }^{4} / T_{\mathrm{RH}} M_{P}^{3}$. We find that a sufficient density of dark matter can be produced over a wide range of dark matter masses: from $1 \mathrm{GeV}$ to $1 \mathrm{ZeV}$.
\end{abstract}

DOI: 10.1103/PhysRevD.103.115009

\section{INTRODUCTION}

While we have considerable certainty in the existence of dark matter (DM), its identity and interactions with the Standard Model are entirely unknown. The lack of a signal in direct detection experiments [1-3] sets strong limits on the DM-proton cross section. Furthermore, the lack of detection at the LHC [4] also seems also to point towards more massive candidates and perhaps a more massive beyond-the-Standard-Model sector than was originally envisioned [5-7].

The mechanism by which dark matter particles populate the Universe is also unknown. Commonly, GeV-TeV DM candidates are assumed to exist in equilibrium as part of the thermal bath. As the temperature falls below the DM mass, they drop out of equilibrium, and their relic density freezes out $[5,8-11]$. However, it is quite possible that DM particles never attain thermal equilibrium. They may either be too massive, or their interactions with the Standard Model may be too weak. For example, particles which interact with the Standard Model primarily through gravity, such as the gravitino, never achieve equilibrium, though they are produced by the thermal bath at reheating after inflation [7,12-14]. Very roughly, their abundance $Y \sim n_{3 / 2} / n_{\gamma}$ can

\footnotetext{
yann.mambrini@th.u-psud.fr

olive@physics.umn.edu
}

Published by the American Physical Society under the terms of the Creative Commons Attribution 4.0 International license. Further distribution of this work must maintain attribution to the author(s) and the published article's title, journal citation, and DOI. Funded by SCOAP. be estimated from their production rate, $Y \sim \Gamma_{p} / H \sim$ $T_{\mathrm{RH}} / M_{P}$, where $H$ is the Hubble parameter, $T_{\mathrm{RH}}$ is the reheating temperature after inflation, and $M_{P}=1 / \sqrt{8 \pi G_{N}}$ is the (reduced) Planck mass. This mechanism, now generally referred to as freeze-in, applies to a wider class of dark matter candidates known as feebly interacting massive particles or FIMPs [15-19]. Other examples include dark matter particles produced by the exchange of a massive $Z^{\prime}$ [20] or massive spin-2 [21].

It is also possible that DM is produced in the decay of the inflaton, either directly [22-25] or radiatively [26]. It has also been observed that annihilation-like processes such as $\phi \phi \rightarrow S S$, where $\phi$ is the inflaton and $S$ is a dark matter scalar, mediated by gravity, can produce a sufficient abundance of dark matter [27]. Indeed, the production of dark matter solely mediated by gravity is a minimal contribution which is nearly model independent, as we discuss in more detail below. The production of dark matter mediated by gravity from the thermal bath is subdominant [21,28-31].

Often, reheating is characterized by a single temperature, $T_{\mathrm{RH}}$, which may be defined when the energy density in the newly produced thermal bath becomes equal to the energy density still stored in inflaton oscillations. However, when one drops the approximation of instantaneous reheating, one finds that initially, the Universe reheats to a potentially much higher temperature, $T_{\max }$, though very little of the total energy density of the Universe is in the form of radiation [23-25,32-34]. For all models in which dark matter is produced during the reheating process after inflation, the dark matter abundance may be sensitive to the evolution of the temperature between $T_{\max }$ and $T_{\mathrm{RH}}$. 
The purpose of this paper is to compute the minimal dark matter abundance produced solely through gravity during reheating, taking into account the sensitivity of the result to details of the reheating process. We will show that the bulk of the production of dark matter occurs at temperatures higher than the reheating temperature (when radiation begins to dominate the energy density) while the radiation bath begins to form. We stress that this process is always present, though other forms of production, including preheating, may also play a role and affect the final dark matter abundance. Here, we will only consider the universal gravitational production channel.

The paper is organized as follows: In the next section, we lay out the framework of our calculation. Starting with the universal coupling of gravity to the stress-energy tensor for either scalars or fermions, we compute the annihilation-like rate for $\phi \phi \rightarrow S S$ (for scalar dark matter) and $\phi \phi \rightarrow \chi \chi$ in the case of fermionic dark matter. In Sec. III, we compute the dark matter abundance based on the detailed process of reheating between $T_{\max }$ and $T_{\mathrm{RH}}$. Then, in Sec. IV, we relate these results to possible inflaton couplings to the Standard Model (which are responsible for reheating), and we draw our conclusions in Sec. V.

\section{THE FRAMEWORK}

The universal interaction that surely exists between the inflaton and any dark sector is gravity. In particular, the $s$ channel exchange of a graviton, shown in Fig. 1, can be obtained from the Lagrangian (see, e.g., Ref. [35])

$$
\mathcal{L}=\frac{1}{2 M_{P}} h_{\mu \nu} T_{\phi}^{\mu \nu}+\frac{1}{2 M_{P}} h_{\mu \nu} T_{S / \chi}^{\mu \nu}
$$

where $h_{\mu \nu}$ is the metric perturbation corresponding to the graviton and we consider either a scalar ${ }^{1} S$ or a fermion $\chi$ as dark matter, whose stress-energy tensors are given by

$$
\begin{gathered}
T_{X=\phi, S}^{\mu \nu}=\partial^{\mu} X \partial^{\nu} X-g^{\mu \nu}\left[\frac{1}{2} \partial^{\alpha} X \partial_{\alpha} X-V(X)\right], \\
T_{\chi}^{\mu \nu}=\frac{i}{4}\left[\bar{\chi} \gamma^{\mu} \stackrel{\leftrightarrow}{\partial^{\nu}} \chi+\bar{\chi} \gamma^{\nu} \stackrel{\leftrightarrow}{\partial^{\mu}} \chi\right]-g^{\mu \nu}\left[\frac{i}{2} \bar{\chi} \gamma^{\alpha} \stackrel{\leftrightarrow}{\partial_{\alpha}} \chi-m_{\chi} \bar{\chi} \chi\right]
\end{gathered}
$$

where $V(X)$ is the scalar potential for either the inflaton or scalar dark matter.

The amplitudes relevant for the computation of the processes $\phi\left(p_{1}\right)+\phi\left(p_{2}\right) \rightarrow \mathrm{DM}^{j}\left(p_{3}\right)+\mathrm{DM}^{j}\left(p_{4}\right)$ can be parametrized by

\footnotetext{
${ }^{1}$ We will consider a real scalar or Dirac fermionic as dark matter. Generalization to a complex scalar or vectorial dark matter is straightforward.
}

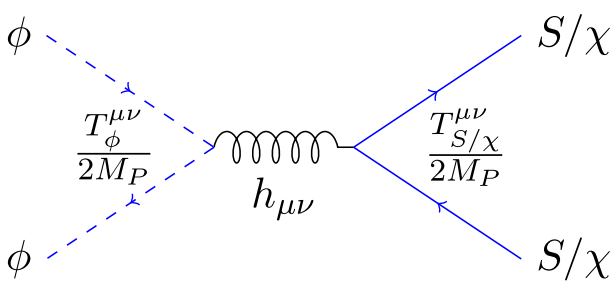

FIG. 1. Illustration of the production of dark matter through the gravitational scattering of the inflaton.

$$
\mathcal{M}^{\phi j} \propto M_{\mu \nu}^{\phi} \Pi^{\mu \nu \rho \sigma} M_{\rho \sigma}^{j}
$$

where $j$ denotes the spin of the DM involved in the process, and $j=0,1 / 2$. $\Pi^{\mu \nu \rho \sigma}$ denotes the propagator of the graviton with momentum $k=p_{1}+p_{2}$ :

$$
\Pi^{\mu \nu \rho \sigma}(k)=\frac{\frac{1}{2} \eta^{\rho \nu} \eta^{\sigma \mu}+\frac{1}{2} \eta^{\rho \mu} \eta^{\sigma \nu}-\frac{1}{2} \eta^{\rho \sigma} \eta^{\mu \nu}}{k^{2}} .
$$

The partial amplitudes, $M_{\mu \nu}^{a}$, can be expressed as

$$
\begin{aligned}
M_{\mu \nu}^{0} & =\frac{1}{2}\left(p_{1 \mu} p_{2 \nu}+p_{1 \nu} p_{2 \mu}-g_{\mu \nu} p_{1} \cdot p_{2}-g_{\mu \nu} m_{\phi}^{2}\right), \\
M_{\mu \nu}^{1 / 2} & =\frac{1}{4} \bar{v}\left(p_{4}\right)\left[\gamma_{\mu}\left(p_{3}-p_{4}\right)_{\nu}+\gamma_{\nu}\left(p_{3}-p_{4}\right)_{\mu}\right] u\left(p_{3}\right),
\end{aligned}
$$

with a similar expression for scalar dark matter in terms of the dark matter momenta, $p_{3}, p_{4}$, and mass $m_{S}^{2}$.

It is then straightforward to compute the production rate of the dark matter. Given the inflaton number density, $n_{\phi}=\rho_{\phi} / m_{\phi}$, the rate for the process depicted in Fig. 1 is

$$
\begin{gathered}
\Gamma_{\phi \phi \rightarrow S S}=\frac{\rho_{\phi} m_{\phi}}{1024 \pi M_{P}^{4}}\left(1+\frac{m_{s}^{2}}{2 m_{\phi}^{2}}\right)^{2} \sqrt{1-\frac{m_{s}^{2}}{m_{\phi}^{2}}}, \\
\Gamma_{\phi \phi \rightarrow \chi \chi}=\frac{\rho_{\phi} m_{\chi}^{2}}{4096 \pi M_{P}^{4} m_{\phi}}\left(1-\frac{m_{\chi}^{2}}{m_{\phi}^{2}}\right)^{3 / 2} .
\end{gathered}
$$

Note the difference in behavior in the expressions for fermionic and scalar dark matter, especially in the mass dependence. On dimensional grounds, both are proportional to $n_{\phi} m_{\phi}^{2} / M_{P}^{4}=\rho_{\phi} m_{\phi} / M_{P}^{4}$. However, our rates in Eqs. (7) and (8) correspond to $s$-wave scattering within the condensate. As a result, in the case of a Dirac fermionantifermion pair in the final state, we require a spin flip leading to a suppression by a factor $\left(m_{\chi} / m_{\phi}\right)^{2}$, making the rate proportional to $\rho_{\phi} m_{\chi}^{2} / m_{\phi} M_{P}^{4}$. A similar expression for the rate producing scalars was found in Ref. [27].

\section{DARK MATTER PRODUCTION}

In many models of inflation, after the period of exponential expansion, the inflaton begins a series of oscillations about a minimum. During the initial stages of 
oscillations, the Universe expands as if it were dominated by nonrelativistic matter. Inflaton decays begin the process of reheating $[12,36]$. While we assume that decay products thermalize rapidly [37], we do not assume that the decay is instantaneous. Instead, we include the effects due to the evolution of the temperature of the radiation bath from its initial creation to a temperature $T_{\max }$ until it begins to dominate the expansion at $T_{\mathrm{RH}}$.

Let us start by considering the evolution of the radiation bath using the conservation equation for radiation (produced by the decay of the inflaton),

$$
\frac{d \rho_{R}}{d t}+4 H \rho_{R}=\Gamma_{\phi} \rho_{\phi},
$$

where $\Gamma_{\phi}$ is the inflaton decay rate, and $\rho_{R}$ and $\rho_{\phi}$ are the energy densities of radiation and the inflaton, respectively. This equation can be rewritten as

$$
\frac{d X}{d a}=\frac{\Gamma_{\phi} \rho_{\phi}}{H} a^{3},
$$

where $X=\rho_{R} a^{4}, a$ is the cosmological scale factor, and $H \equiv \dot{a} / a \approx \sqrt{\rho_{\phi}} / \sqrt{3} M_{P}$ up to the epoch of radiation domination. Assuming inflaton oscillations about a quadratic potential, ${ }^{2} \rho_{\phi}=\rho_{e}\left(a_{e} / a\right)^{3}$, where $\rho_{e}$ is the energy density stored by the inflaton at the end of the inflationary phase, and Eq. (10) is easily integrated to give

$$
\rho_{R}=\frac{2 \sqrt{3}}{5} \Gamma_{\phi} M_{P} \sqrt{\rho_{e}}\left(\frac{a_{e}}{a}\right)^{3 / 2}\left[1-\left(\frac{a_{e}}{a}\right)^{5 / 2}\right]=\alpha T^{4},
$$

where $\alpha=g_{T} \pi^{2} / 30$ and $g_{T}$ is the number of relativistic degrees of freedom, at temperature $T$. Thus, in the case of noninstantaneous reheating, when $a \gg a_{e}$, and as long as the inflaton dominates the energy density, the temperature of the radiation bath falls as $T \propto a^{-3 / 8}$ as energy is injected into the bath from inflaton decay [24,26,32]. For $a \gg a_{e}$, with the help of Eq. (11), we can then write

$$
H(T) \simeq \frac{\sqrt{\rho_{\phi}}}{\sqrt{3} M_{P}}=\frac{5}{6} \frac{\alpha}{\Gamma_{\phi} M_{P}^{2}} T^{4} .
$$

Within the same limit, we can parametrize the reheating temperature by

$$
T_{\mathrm{RH}}=\left(\frac{4}{3 \alpha c^{2}}\right)^{1 / 4}\left(\Gamma_{\phi} M_{P}\right)^{1 / 2},
$$

where $c=1$ if we define the reheat temperature by $t_{\mathrm{RH}}^{-1}=\frac{3}{2} H=\Gamma_{\phi}$, and $c=5 / 3$ if we define $T_{\mathrm{RH}}$ by

\footnotetext{
${ }^{2}$ For similar results about a more general potential, see Ref. [38].
}

$\rho_{\phi}\left(T_{\mathrm{RH}}\right)=\rho_{R}\left(T_{\mathrm{RH}}\right)$. Here, we have chosen the latter, which can easily be derived by rewriting Eq. (11) in the limit $a \gg a_{e}$ :

$$
\rho_{R} \simeq \frac{2 \sqrt{3}}{5} \Gamma_{\phi} M_{P} \sqrt{\rho_{\phi}\left(T_{\mathrm{RH}}\right)}\left(\frac{a_{\mathrm{RH}}}{a}\right)^{3 / 2},
$$

so that $\rho_{\phi}\left(T_{\mathrm{RH}}\right)=\rho_{R}\left(T_{\mathrm{RH}}\right)$ implies that

$$
\rho_{R}\left(T_{\mathrm{RH}}\right)=\alpha T_{\mathrm{RH}}^{4} \simeq \frac{12}{25} \Gamma_{\phi}^{2} M_{P}^{2}
$$

Then, the expression for $H(T)$ in a Universe dominated by the inflaton is given by $[26,38]$

$$
H(T)=\sqrt{\frac{\alpha}{3}} \frac{T^{4}}{T_{\mathrm{RH}}^{2} M_{P}}
$$

and

$$
\rho_{\phi}=\rho_{R}\left(T_{\mathrm{RH}}\right)\left(\frac{a_{\mathrm{RH}}}{a}\right)^{3}=\alpha \frac{T^{8}}{T_{\mathrm{RH}}^{4}} .
$$

When the limit $a \gg a_{e}$ cannot be applied, the reheating temperature is the solution of the equation

$$
\sqrt{\rho_{\mathrm{RH}}}=\alpha^{1 / 2} T_{\mathrm{RH}}^{2}=\frac{2 \sqrt{3}}{5} \Gamma_{\phi} M_{P}\left[1-\left(\frac{\rho_{\mathrm{RH}}}{\rho_{e}}\right)^{5 / 6}\right],
$$

where $\rho_{\mathrm{RH}}=\rho_{\phi}\left(T_{R H}\right)=\rho_{R}\left(T_{\mathrm{RH}}\right)$.

Note that the maximum temperature attained, $T_{\max }$, can be found from the extremum of Eq. (11) with respect to $a$. We obtain

$$
\frac{a_{\max }}{a_{e}}=\left(\frac{8}{3}\right)^{2 / 5}
$$

implying

$$
\rho_{R}\left(a_{\max }\right)=\frac{\sqrt{3}}{4} \Gamma_{\phi} M_{P} \sqrt{\rho_{e}}\left(\frac{3}{8}\right)^{3 / 5}=\alpha T_{\max }^{4} .
$$

Combining Eqs. (18) and (20) gives

$$
\left(\frac{T_{\max }}{T_{\mathrm{RH}}}\right)^{4}=\frac{25 \sqrt{\rho_{e}}}{16 \sqrt{3} \Gamma_{\phi} M_{P}}\left(\frac{3}{8}\right)^{3 / 5}\left[1-\left(\frac{a_{e}}{a_{\mathrm{RH}}}\right)^{5 / 2}\right]^{-2} .
$$

The relic abundance of dark matter is obtained by solving the Boltzmann equation for the number density of dark matter particles: 


$$
\frac{d n_{j}}{d t}+3 H n_{j}=R_{j}(T)
$$

Writing $Y_{j}=n_{j} a^{3}$, Eq. (22) can be simplified to

$$
\frac{d Y_{j}}{d a}=\frac{R_{j}(T) a^{2}}{H}
$$

where $R_{j}(T)=\frac{\rho_{\phi}}{m_{\phi}} \Gamma_{\phi \phi \rightarrow j j}$ is the production rate (per unit volume and unit time), and the yield $Y_{j}$ is proportional to the number of dark matter quanta produced in the comoving frame. It is important to note that because $R_{j} \propto \rho_{\phi}^{2} \propto T^{16}$, there is a very strong temperature dependence in Eq. (23), making the result sensitive to the maximum temperature attained.

Using Eq. (17), it is straightforward to integrate Eq. (23) between $a_{e}$ and $a_{\mathrm{RH}}$. We find

$$
Y_{j}\left(T_{\mathrm{RH}}\right)=\frac{2 \gamma_{j} M_{P}}{\sqrt{3} m_{\phi}} \rho_{e}^{3 / 2} a_{e}^{3}\left[1-\left(\frac{a_{e}}{a_{\mathrm{RH}}}\right)^{3 / 2}\right],
$$

where $\gamma_{j}=\Gamma_{\phi \phi \rightarrow j j} / \rho_{\phi}$. The yield can also be written in terms of $\rho_{R}$, and the number density becomes

$$
n_{j}\left(T_{\mathrm{RH}}\right)=\frac{2 \gamma_{j} M_{P}}{\sqrt{3} m_{\phi}} \alpha^{3 / 2} T_{\mathrm{RH}}^{6}\left[\left(\frac{a_{\mathrm{RH}}}{a_{e}}\right)^{3 / 2}-1\right] .
$$

Noting that we can write $\left(a_{e} / a_{\mathrm{RH}}\right)=$ $\left(a_{e} / a_{\max }\right)\left(a_{\max } / a_{\mathrm{RH}}\right)=(3 / 8)^{2 / 5}\left(T_{\max } / T_{\mathrm{RH}}\right)^{8 / 3}$, the density can be expressed as

$$
n_{j}\left(T_{\mathrm{RH}}\right)=\frac{2 \gamma_{j} M_{P}}{\sqrt{3} m_{\phi}} \alpha^{3 / 2} T_{\mathrm{RH}}^{2}\left[\left(\frac{8}{3}\right)^{3 / 5} T_{\mathrm{max}}^{4}-T_{\mathrm{RH}}^{4}\right] .
$$

Equation (26) is valid so long as $a_{\mathrm{RH}}>a_{\max }$. For sufficiently large $\Gamma_{\phi}$, the radiation energy density will equal the inflaton oscillation energy density when $T=T_{\max }$, and thus $T_{\mathrm{RH}}=T_{\max }$. At larger $\Gamma_{\phi}$, we can write

$$
n_{j}\left(T_{\mathrm{RH}}\right)=\frac{2 \gamma_{j} M_{P}}{\sqrt{3} m_{\phi}} \alpha^{3 / 2} T_{\mathrm{RH}}^{6}\left[\left(\frac{\sqrt{\rho_{e}}}{\alpha^{1 / 2} T_{\mathrm{RH}}^{2}}\right)-1\right],
$$

as the temperature $T_{\max }$ is never attained.

The fraction of critical density in dark matter can be written as

$$
\Omega_{j} h^{2}=1.6 \times 10^{8}\left(\frac{g_{0}}{g_{\mathrm{RH}}}\right)\left(\frac{n_{j}\left(T_{\mathrm{RH}}\right)}{T_{\mathrm{RH}}^{3}}\right)\left(\frac{m_{j}}{1 \mathrm{GeV}}\right),
$$

where the numerical factor is $\pi^{2} n_{\gamma}\left(T_{0}\right) / 2 \zeta(3) \rho_{c}$, $g_{0}=43 / 11, g_{\mathrm{RH}}=427 / 4$, and $n_{j}\left(T_{\mathrm{RH}}\right)$ is obtained from either Eq. (26) or Eq. (27) for $a_{\mathrm{RH}}>a_{\max }$ or $a_{\mathrm{RH}}<a_{\max }$, respectively. We finally obtain

$$
\begin{aligned}
\frac{\Omega_{s} h^{2}}{0.1} \simeq & \left(\frac{T_{\mathrm{RH}}}{10^{10}}\right)^{3}\left(\frac{T_{\max } / T_{\mathrm{RH}}}{100}\right)^{4}\left(\frac{m_{S}}{1.75 \times 10^{10}}\right) \\
& \times\left(1+\frac{m_{s}^{2}}{2 m_{\phi}^{2}}\right)^{2} \sqrt{1-\frac{m_{s}^{2}}{m_{\phi}^{2}} ; a_{\mathrm{RH}}>a_{\max },} \\
\frac{\Omega_{s} h^{2}}{0.1} & \simeq\left(\frac{T_{\mathrm{RH}}}{10^{10}}\right) \sqrt{\frac{\rho_{e}}{10^{60}}}\left(\frac{m_{S}}{1.9 \times 10^{9}}\right) \\
& \times\left(1+\frac{m_{s}^{2}}{2 m_{\phi}^{2}}\right)^{2} \sqrt{1-\frac{m_{s}^{2}}{m_{\phi}^{2}}} ; a_{\mathrm{RH}}<a_{\max }
\end{aligned}
$$

for scalar dark matter and

$$
\begin{aligned}
\frac{\Omega_{\chi} h^{2}}{0.1} \simeq & \left(\frac{T_{\mathrm{RH}}}{10^{10}}\right)^{3}\left(\frac{T_{\mathrm{max}} / T_{\mathrm{RH}}}{100}\right)^{4}\left(\frac{m_{\chi}}{4.0 \times 10^{12}}\right)^{3} \\
& \times\left(\frac{3 \times 10^{13}}{m_{\phi}}\right)^{2}\left(1-\frac{m_{\chi}^{2}}{m_{\phi}^{2}}\right)^{3 / 2} ; a_{\mathrm{RH}}>a_{\max }, \\
\frac{\Omega_{\chi} h^{2}}{0.1} \simeq & \left(\frac{T_{\mathrm{RH}}}{10^{10}}\right) \sqrt{\frac{\rho_{e}}{10^{60}}}\left(\frac{m_{\chi}}{1.9 \times 10^{12}}\right)^{3} \\
& \times\left(\frac{3 \times 10^{13}}{m_{\phi}}\right)^{2}\left(1-\frac{m_{\chi}^{2}}{m_{\phi}^{2}}\right)^{3 / 2} ; a_{\mathrm{RH}}<a_{\max }
\end{aligned}
$$

for fermionic dark matter. Units of energy are given in $\mathrm{GeV}$ (and $\mathrm{GeV}^{4}$ for $\rho_{e}$ ). Note that these expressions are approximations which are valid so long as $a_{\mathrm{RH}}$ is not very close to either $a_{\max }$ or $a_{e}$. More generally, the relic density is obtained from Eq. (28) with either Eq. (26) or Eq. (27). As expected, there is a stronger dependence of the relic abundance on the mass of the dark matter in the fermionic case due to its production rate.

We show in Fig. 2 the allowed parameter space in the ( $\left.m_{S, \chi}, T_{\mathrm{RH}}\right)$ plane for scalar (blue, dashed) and fermionic (red, solid) dark matter for several values of $T_{\max } / T_{\mathrm{RH}}$. The lines correspond to values of $\Omega h^{2}=0.12$ [39] for each choice of $T_{\max } / T_{\mathrm{RH}}$. In each case, $a_{\mathrm{RH}}>a_{\max }$. All points above the lines are excluded because they lead to an overabundance. We see, for example, that $\mathrm{GeV}-\mathrm{ZeV}$ dark matter can be obtained with reasonable values of $T_{\mathrm{RH}}$ and $T_{\text {max }}$ by pure gravitational production through inflaton scattering. We also see that due to the additional mass suppression $\left(m_{\chi} / m_{\phi}\right)^{2}$, for fermionic DM, it is necessary to consider higher fermionic masses $(\gtrsim 100 \mathrm{PeV}$ for $\left.T_{\text {max }} / T_{\mathrm{RH}}<1000\right)$ to achieve $\Omega_{\chi} h^{2}=0.12$. Note that we have assumed $m_{\phi}=3 \times 10^{13} \mathrm{GeV}$ in Fig. 2 and the kinematic suppression when $m_{s} \geq m_{\phi}$ would appear to the right of the region plotted.

\section{COUPLING TO THE STANDARD MODEL}

In the previous section, we concentrated on results for a given reheating and maximum temperature reached by the 


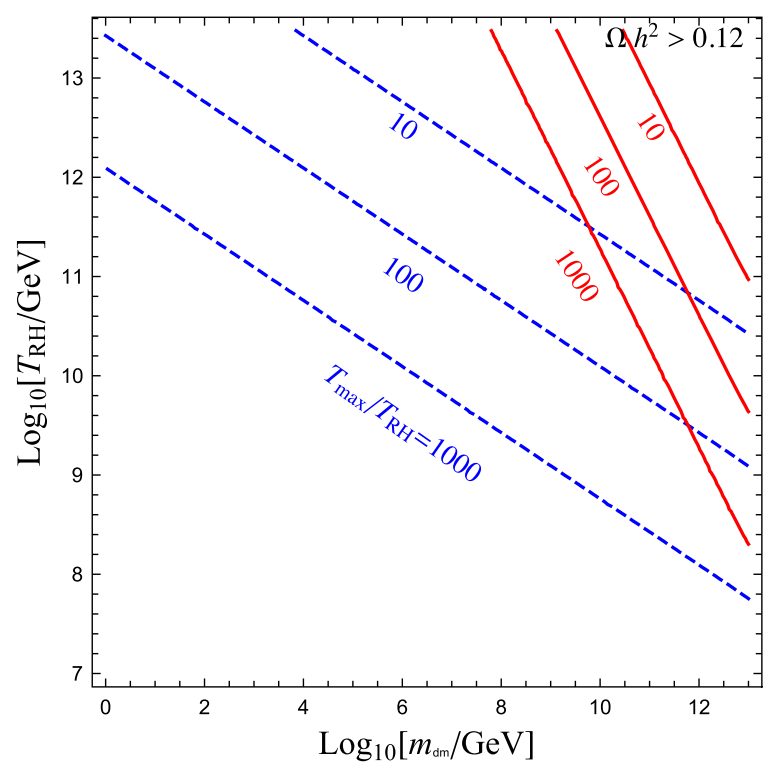

FIG. 2. Points yielding the Planck relic density $\Omega_{S, \chi} h^{2}=0.12$ for scalar (blue, dashed) and fermionic (red, solid) dark matter, in the $\left(m_{S, \chi}, T_{\mathrm{RH}}\right)$ plane for several values of $T_{\max } / T_{\mathrm{RH}}$ as labeled.

thermal bath during the reheating process. In any concrete model of inflation, these temperatures can be traced to two quantities: the coupling of the inflaton to the Standard Model, which ultimately determines the reheating temperature, and the initial energy density of the inflaton at the end of the inflationary phase, which allows one to determine the amount of energy available for the thermal bath, and hence the maximum temperature. If one supposes a simple effective coupling of the inflaton to the Standard Model fermions of the type

$$
\mathcal{L}_{\phi-S M}^{y}=y \phi \bar{f} f
$$

leading to

$$
\Gamma_{\phi}=\frac{y^{2}}{8 \pi} m_{\phi}
$$

and using Eq. (21), we can write the ratio

$$
\left(\frac{T_{\max }}{T_{\mathrm{RH}}}\right)^{4}=\frac{25 \pi \sqrt{\rho_{e}}}{2 \sqrt{3} y^{2} m_{\phi} M_{P}}\left(\frac{3}{8}\right)^{3 / 5}\left[1-\left(\frac{a_{e}}{a_{\mathrm{RH}}}\right)^{5 / 2}\right]^{-2} .
$$

In models which are dominated by a quadratic term after inflation, we expect $\rho_{e} \sim m_{\phi}^{2} M_{P}^{2}$, and for example, in the Starobinsky model of inflation [40], $\rho_{e}^{1 / 4} \sim$ $0.65 m_{\phi}^{1 / 2} M_{P}^{1 / 2}=5.5 \times 10^{15} \mathrm{GeV}$ [41]. For sufficiently large coupling, $y \simeq .67\left(\rho_{e}^{1 / 4} / 10^{15} \mathrm{GeV}\right), T_{\max } / T_{\mathrm{RH}}=1$, and reheating is nearly instantaneous. If reheating is primarily due to a coupling of the inflaton to bosons,
$\mathcal{L}_{\phi-S M}^{\mu}=\mu \phi b b$, equivalent results are obtained by substituting $y \rightarrow \mu / m_{\phi}$.

Similarly, we can also compute $T_{\mathrm{RH}}$ by combining Eqs. (18) and (33):

$$
T_{\mathrm{RH}}^{4}=\left(\frac{3 y^{4}}{400 \alpha \pi^{2}}\right) m_{\phi}^{2} M_{P}^{2}\left[1-\left(\frac{\alpha T_{\mathrm{RH}}^{4}}{\rho_{e}}\right)^{5 / 6}\right]^{2} .
$$

For sufficiently large $\rho_{e}$, the second term in the bracket can be neglected; otherwise, $T_{\mathrm{RH}}$ can be solved for iteratively. We can now express the relic density in terms of the coupling $y$ using Eqs. (35) and (36) in Eqs. (26) and (27). When $a_{\mathrm{RH}} \gg a_{\max }$, we can write relatively compact expressions by combining Eqs. (29), (31), (35), and (36), and one obtains for $m_{S, \chi} \ll m_{\phi}$

$$
\begin{gathered}
\frac{\Omega_{S} h^{2}}{0.1}=\frac{y}{10^{-7}} \sqrt{\frac{\rho_{e}}{10^{64}}} \sqrt{\frac{3 \times 10^{13}}{m_{\phi}}} \frac{m_{S}}{5.2 \times 10^{9}}, \\
\frac{\Omega_{\chi} h^{2}}{0.1}=\frac{y}{10^{-7}} \sqrt{\frac{\rho_{e}}{10^{64}}}\left(\frac{m_{\chi}}{2.6 \times 10^{12}}\right)^{3}\left(\frac{3 \times 10^{13}}{m_{\phi}}\right)^{3 / 2},
\end{gathered}
$$

where as before, dimensions for mass are in $\mathrm{GeV}$, with $\mathrm{GeV}^{4}$ for $\rho_{e}$. We see that for a given Standard Model coupling $\left(y=10^{-7}\right.$ for example), the dark matter mass needed to reach a reasonable relic abundance is much higher in the fermionic case than in the scalar case, bearing in mind the minimality of the model being considered.

We plot in Fig. 3 the required relation between $m_{S, \chi}$ and $y$ to obtain $\Omega_{S, \chi} h^{2}=0.12$. Values of $\Omega h^{2}>0.12$ are

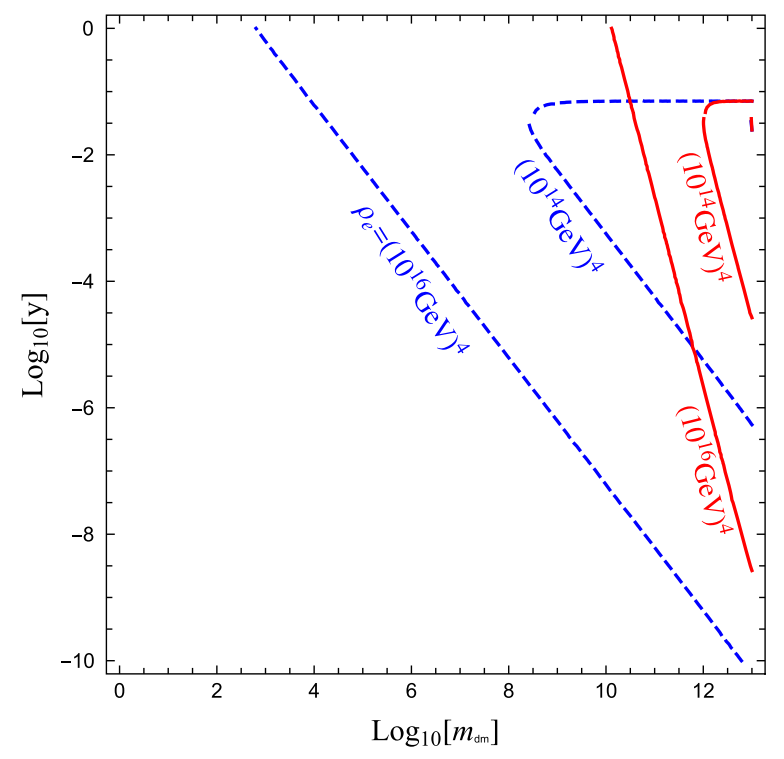

FIG. 3. Points in the $\left(m_{S, \chi}, y\right)$ plane yielding the Planckdetermined relic density $\Omega_{S, \chi} h^{2}=0.12$ in the case of a scalar dark matter (blue, dashed) and a fermionic dark matter (red, solid) for two values of $\rho_{e}$ as labeled. 
obtained for points to the right of the negatively sloped lines and below the horizontal lines for $\rho_{e}^{1 / 4}=10^{14} \mathrm{GeV}$. Note that for $\rho_{e}^{1 / 4}=10^{14} \mathrm{GeV}$, the curves begin to bend when the second term in the bracket in Eq. (35) approaches 1. For small values of $y, T_{\max } \gg T_{\mathrm{RH}}$, and the relic density in Eq. (29) scales as $T_{\mathrm{RH}}^{3}\left(T_{\max } / T_{\mathrm{RH}}\right)^{4} m_{s}$. Since $\left(T_{\mathrm{RH}}\right)^{3} \sim y^{3}, \quad\left(T_{\max } / T_{\mathrm{RH}}\right)^{4} \sim y^{-2}$, we see that $y \sim m_{S}^{-1}$ for fixed relic density. At larger values of $y$, when $a_{\mathrm{RH}}<a_{\mathrm{max}}$, the density in Eq. (30) scales as $T_{\mathrm{RH}} m_{s}$. However, we can see from Eq. (36), that $T_{\mathrm{RH}}$ reaches its maximal value when $\alpha T_{\mathrm{RH}}^{4} \lesssim \rho_{e}$. At these values of $y$ [which are not much larger than $.67\left(\rho_{e}^{1 / 4} / 10^{15} \mathrm{GeV}\right)$ - the value needed to make $T_{\mathrm{RH}}=T_{\max }$, the number density obtained from Eq. (27) begins to decrease. Thus, a fixed energy density which is proportional to $n_{j} m_{j}$ requires a rapidly larger value of the dark matter mass. This accounts for the curve bending to the horizontal at large values of $m_{j}$. Physically, at these values of $y$, the rate of inflaton decay exceeds the rate of dark matter production from scattering, and production through the gravitational process ceases. For $\rho_{e}^{1 / 4}>1.5 \times 10^{15} \mathrm{GeV}$, this behavior occurs only at $y>1$. Once again, the region with kinematic suppression lies to the right of the region shown in the figure.

It is also possible that inflaton scattering into StandardModel scalars affects the reheating process, and the value of $T_{\max }$ in particular [38]. For example, a Lagrangian contribution of

$$
\mathcal{L}_{\phi-S M}^{\sigma}=\sigma \phi^{2} b^{2}
$$

where $b$ represents a Standard-Model boson. In this case, one still requires the Yukawa coupling [Eq. (33)] to complete the reheating process (so that the energy density in radiation comes to dominate over the energy density in $\phi$ ) [38]. The interaction generated by Eq. (39) can alter the maximum temperature $T_{\max }$ for sufficiently large values of $\sigma$. In this case,

$$
\left(\frac{T_{\max }}{T_{\mathrm{RH}}}\right)^{4}=\frac{6400 \pi \sigma^{2} \rho_{e}^{3 / 2}}{\sqrt{3} y^{4} m_{\phi}^{5} M_{P}}\left(\frac{2}{3}\right)^{18},
$$

giving

$$
\begin{aligned}
\frac{\Omega_{S} h^{2}}{0.1}= & \frac{10^{-6}}{y}\left(\frac{\sigma}{10^{-9}}\right)^{2}\left(\frac{\rho_{e}}{\left(10^{16}\right)^{4}}\right)^{3 / 2}\left(\frac{3 \times 10^{13}}{m_{\phi}}\right)^{7 / 2} \\
& \times\left(\frac{m_{S}}{1.2 \times 10^{5}}\right)
\end{aligned}
$$

and

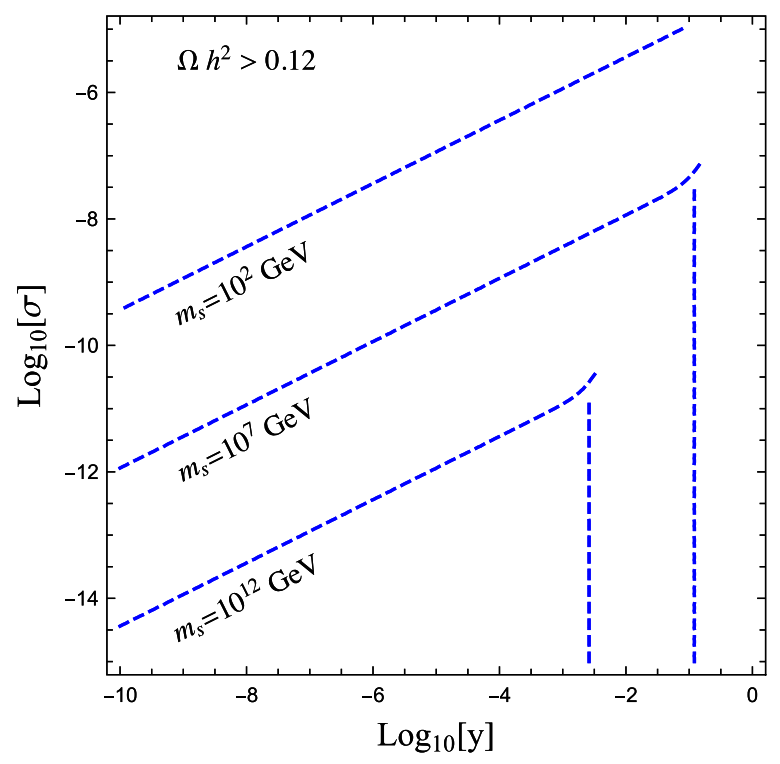

FIG. 4. Points respecting Planck constraint $\Omega_{S} h^{2}=0.12$ in the case of scalar dark matter, in the plane $(y, \sigma)$ for different values of $m_{S}$.

$$
\begin{aligned}
\frac{\Omega_{\chi} h^{2}}{0.1}= & \frac{10^{-6}}{y}\left(\frac{\sigma}{10^{-9}}\right)^{2}\left(\frac{\rho_{e}}{\left(10^{16}\right)^{4}}\right)^{3 / 2}\left(\frac{m_{\chi}}{7.6 \times 10^{10}}\right)^{3} \\
& \times\left(\frac{3 \times 10^{13}}{m_{\phi}}\right)^{11 / 2} .
\end{aligned}
$$

Note that for a given value of $\sigma$, the relic density increases with decreasing $y$. This is valid only so long as $T_{\max }>T_{\mathrm{RH}}$. The limiting value of $y$ is found by setting Eq. (40) equal to 1.

We show in Fig. 4 the parameter space allowed in the plane $(y, \sigma)$ in the case of scalar dark matter for different values of its mass $m_{\chi}$. Note that it is possible to have quite low values of $y$ (and thus $T_{\mathrm{RH}}$ ), while still being able to produce dark matter in sufficient amounts due to the value of $\sigma$ generating a high maximum temperature $T_{\max }$, and thus a large production rate. The maximum value for $y$ occurs when $T_{\max }=T_{\mathrm{RH}}$ in Eq. (40), where the relic abundance depends only on $T_{\mathrm{RH}}$ (and is thus independent of $\sigma$ ), as we can see in the plot. The same curves for fermionic dark matter are obtained for $m_{\chi}=1.5 \times 10^{9}\left(m_{S} / \mathrm{GeV}\right)^{1 / 3}$.

\section{CONCLUSIONS}

We have derived the conditions for producing sufficient dark matter from inflaton scattering during reheating by $s$-channel graviton exchange. This process is always present, independent of the model of inflation. We have shown that the final abundance of dark matter depends not only on the reheating temperature, but also on the maximum temperature, and hence on the detailed evolution of the reheating process. During the exit from exponential expansion, many models of inflation begin a period of oscillations leading to reheating. At the onset of these oscillations, the inflaton 
density is high, and the leading contribution to dark matter production occurs at the start of reheating at $T_{\max }$. This represents an absolute minimal amount of dark matter production, and it contributes independently of any interactions the dark matter may have with the Standard Model (or another dark sector if present).

\section{ACKNOWLEDGMENTS}

This work was made possible by Institut Pascal at Universite Paris-Saclay with the support of the P2I research departments and the P2IO Laboratory of Excellence (program "Investissements d'avenir" No. ANR-11-IDEX0003-01 Paris-Saclay and No. ANR-10-LABX-0038). This project has received support from the European Union's Horizon 2020 research and innovation program under the Marie Skłodowska-Curie Grant Agreement No. 860881-HIDDeN and the CNRS PICS MicroDark. The work of K. A. O. was supported in part by DOE Grant No. DE-SC0011842 at the University of Minnesota.
[1] E. Aprile et al. (XENON Collaboration), Phys. Rev. Lett. 121, 111302 (2018).

[2] D. S. Akerib et al. (LUX Collaboration), Phys. Rev. Lett. 118, 021303 (2017).

[3] X. Cui et al. (PandaX-II Collaboration), Phys. Rev. Lett. 119, 181302 (2017).

[4] G. Aad et al. (ATLAS Collaboration), J. High Energy Phys. 09 (2014) 176; 10 (2015) 054; Phys. Rev. D 94, 032003 (2016); S. Chatrchyan et al. (CMS Collaboration), J. High Energy Phys. 06 (2014) 055; V. Khachatryan et al. (CMS Collaboration), J. High Energy Phys. 10 (2016) 006.

[5] P. Hut, Phys. Lett. 69B, 85 (1977); B. W. Lee and S. Weinberg, Phys. Rev. Lett. 39, 165 (1977); M. I. Vysotsky, A. D. Dolgov, and Ya. B. Zeldovich, JETP Lett. 26, 188 (1977), http://jetpletters.ru/ps/1375/article_20858.shtml.

[6] J. Gunn, B. Lee, I. Lerche, D. Schramm, and G. Steigman, Astrophys. J. 223, 1015 (1978).

[7] J. R. Ellis, J. S. Hagelin, D. V. Nanopoulos, K. A. Olive, and M. Srednicki, Nucl. Phys. B238, 453 (1984).

[8] M. Srednicki, R. Watkins, and K. A. Olive, Nucl. Phys. B310, 693 (1988).

[9] P. Gondolo and G. Gelmini, Nucl. Phys. B360, 145 (1991).

[10] K. Griest and D. Seckel, Phys. Rev. D 43, 3191 (1991).

[11] G. Arcadi, M. Dutra, P. Ghosh, M. Lindner, Y. Mambrini, M. Pierre, S. Profumo, and F. S. Queiroz, Eur. Phys. J. C 78, 203 (2018).

[12] D. V. Nanopoulos, K. A. Olive, and M. Srednicki, Phys. Lett. 127B, 30 (1983).

[13] M. Y. Khlopov and A. D. Linde, Phys. Lett. 138B, 265 (1984).

[14] K. A. Olive, D. N. Schramm, and M. Srednicki, Nucl. Phys. B255, 495 (1985).

[15] L. J. Hall, K. Jedamzik, J. March-Russell, and S. M. West, J. High Energy Phys. 03 (2010) 080; X. Chu, T. Hambye, and M. H. G. Tytgat, J. Cosmol. Astropart. Phys. 05 (2012) 034; X. Chu, Y. Mambrini, J. Quevillon, and B. Zaldivar, J. Cosmol. Astropart. Phys. 01 (2014) 034; A. Biswas, D. Borah, and A. Dasgupta, Phys. Rev. D 99, 015033 (2019).

[16] N. Bernal, J. Cosmol. Astropart. Phys. 10 (2020) 006; N. Bernal, J. Rubio, and H. Veermäe, J. Cosmol. Astropart. Phys. 10 (2020) 021.
[17] N. Bernal, M. Heikinheimo, T. Tenkanen, K. Tuominen, and V. Vaskonen, Int. J. Mod. Phys. A 32, 1730023 (2017).

[18] N. Bernal, F. Elahi, C. Maldonado, and J. Unwin, J. Cosmol. Astropart. Phys. 11 (2019) 026.

[19] B. Barman, D. Borah, and R. Roshan, J. Cosmol. Astropart. Phys. 11 (2020) 021.

[20] G. Bhattacharyya, M. Dutra, Y. Mambrini, and M. Pierre, Phys. Rev. D 98, 035038 (2018); A. Banerjee, G. Bhattacharyya, D. Chowdhury, and Y. Mambrini, J. Cosmol. Astropart. Phys. 12 (2019) 009.

[21] N. Bernal, M. Dutra, Y. Mambrini, K. Olive, M. Peloso, and M. Pierre, Phys. Rev. D 97, 115020 (2018).

[22] J. Ellis, M. A. G. Garcia, D. V. Nanopoulos, K. A. Olive, and M. Peloso, J. Cosmol. Astropart. Phys. 03 (2016) 008.

[23] E. Dudas, Y. Mambrini, and K. Olive, Phys. Rev. Lett. 119, 051801 (2017).

[24] M. A. G. Garcia, Y. Mambrini, K. A. Olive, and M. Peloso, Phys. Rev. D 96, 103510 (2017).

[25] M. A. G. Garcia, K. Kaneta, Y. Mambrini, and K. A. Olive, Phys. Rev. D 101, 123507 (2020).

[26] K. Kaneta, Y. Mambrini, and K. A. Olive, Phys. Rev. D 99, 063508 (2019).

[27] Y. Ema, R. Jinno, K. Mukaida, and K. Nakayama, J. Cosmol. Astropart. Phys. 05 (2015) 038; Phys. Rev. D 94, 063517 (2016); Y. Ema, K. Nakayama, and Y. Tang, J. High Energy Phys. 09 (2018) 135.

[28] M. Garny, M. Sandora, and M. S. Sloth, Phys. Rev. Lett. 116, 101302 (2016); M. Garny, A. Palessandro, M. Sandora, and M. S. Sloth, J. Cosmol. Astropart. Phys. 02 (2018) 027.

[29] Y. Tang and Y. L. Wu, Phys. Lett. B 774, 676 (2017).

[30] M. Chianese, B. Fu, and S. F. King, J. Cosmol. Astropart. Phys. 06 (2020) 019; 01 (2021) 034.

[31] M. Redi, A. Tesi, and H. Tillim, J. High Energy Phys. 05 (2021) 010.

[32] G. F. Giudice, E. W. Kolb, and A. Riotto, Phys. Rev. D 64, 023508 (2001); D. J. H. Chung, E. W. Kolb, and A. Riotto, Phys. Rev. D 60, 063504 (1999).

[33] S. L. Chen and Z. Kang, J. Cosmol. Astropart. Phys. 05 (2018) 036.

[34] N. Bernal, J. Cosmol. Astropart. Phys. 10 (2020) 006; A. Di Marco and G. Pradisi, arXiv:2102.00326.

[35] B. R. Holstein, Am. J. Phys. 74, 1002 (2006). 
[36] A. Dolgov and A. D. Linde, Phys. Lett. 116B, 329 (1982); L. Abbott, E. Farhi, and M. B. Wise, Phys. Lett. 117B, 29 (1982).

[37] S. Davidson and S. Sarkar, J. High Energy Phys. 11 (2000) 012; K. Harigaya, K. Mukaida, and M. Yamada, J. High Energy Phys. 07 (2019) 059; K. Harigaya, M. Kawasaki, K. Mukaida, and M. Yamada, Phys. Rev. D 89, 083532 (2014); K. Harigaya and K. Mukaida, J. High Energy Phys. 05 (2014) 006; K. Mukaida and M. Yamada, J. Cosmol.
Astropart. Phys. 02 (2016) 003; M. A. G. Garcia and M. A. Amin, Phys. Rev. D 98, 103504 (2018).

[38] M. A. G. Garcia, K. Kaneta, Y. Mambrini, and K. A. Olive, J. Cosmol. Astropart. Phys. 04 (2021) 012.

[39] N. Aghanim et al. (Planck Collaboration), Astron. Astrophys. 641, A6 (2020).

[40] A. A. Starobinsky, Phys. Lett. 91B, 99 (1980).

[41] J. Ellis, M. A. G. Garcia, D. V. Nanopoulos, and K. A. Olive, J. Cosmol. Astropart. Phys. 07 (2015) 050. 